\section{Welcome}

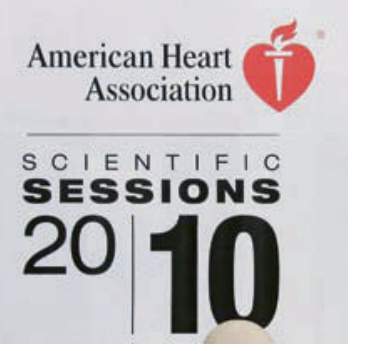

\title{
Renale Denervierung besiegt therapierefraktären Bluthochdruck
}

\begin{abstract}
Zunächst therapieresistente Hypertonie ist doch besiegbar: Bei Patienten, bei denen ein stark erhöhter Blutdruck medikamentös kaum noch zu beeinflussen ist, bietet ein kathetergestütztes Ablationsverfahren die Möglichkeit, den Blutdruck anhaltenden zu senken.
\end{abstract}

\begin{abstract}
Dieses neue endovaskuläre Verfahren ist jetzt erstmals erfolgreich in einer randomisierten kontrollierten Multicenterstudie getestet worden, deren Ergebnisse Studienleiter Dr. Murray Esler aus Melbourne beim AHA in Chicago präsentiert hat.

Die Methode schaltet selektiv die Aktivität renaler sympathischer Nerven aus, die an der Entwicklung und Aufrechterhaltung von Hypertonie mitbeteiligt sind. Afferente und efferente sympathische Nerven verlaufen in der Adventitia der Nierenarterien. Ihre Denervation erfolgt endovas-
\end{abstract}
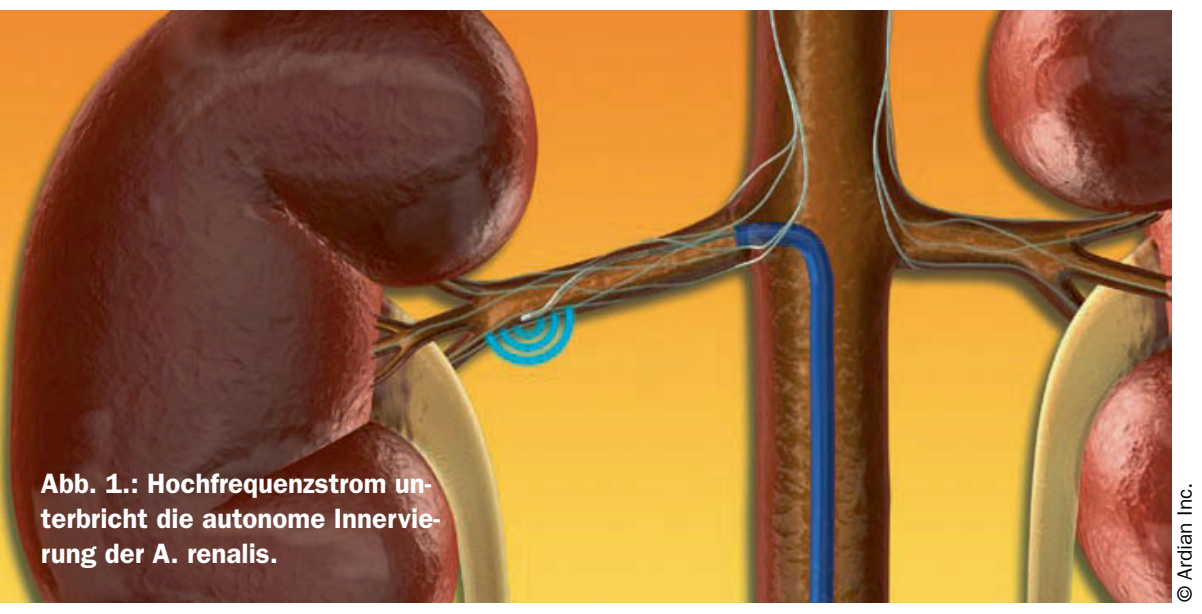

kulär durch kathetergestützte Hochfrequenzablation (s. Abb. 1). Der relativ einfache Eingriff dauert ca. 30-60 Minuten. Die Patienten erhalten zuvor eine lokale Betäubung und ein Schmerzmittel. Es waren auch vier deutsche Zentren beteiligt, etwa die Klinik für Kardiologie des Uniklinikums des Saarlandes, in der nach eigenen Angaben bisher die meisten mit der neuen Methode behandelt wurden.

Insgesamt 106 Hypertoniker, deren systolischer Blutdruck trotz Therapie mit drei oder mehr Antihypertensiva - im Schnitt waren es fünf - deutlich erhöht war (Mittel: $178 \mathrm{mmHg}$ ), nahmen Studie teil. Davon erhielten 49 die Ablation, die Übrigen bildeten die Kontrolle. Die Blutdruckausgangswerte waren in beiden Gruppen fast gleich. Im Vergleich zur Kontrollgruppe, in der es im Schnitt fast keine Veränderung gab, war nach sechs Monaten in der Ablationsgruppe eine signifikante Senkung des in der Praxis gemessenen Drucks um 33/11 mmHg zu verzeichnen. Parallel dazu wurde auch bei der häuslichen Druckmessung $(-22 / 12 \mathrm{mmHg})$ und bei der ambulanten 24-Stundenmessung $(-11 / 7 \mathrm{mmHg})$ eine signifikante Senkung des Blutdrucks festgestellt.

Eine Reduktion des systolischen Drucks um zumindest $10 \mathrm{mmHg}$ hatten nach Ablation 84\%, in der Kontrolle 35\%. Schwere Komplikationen oder unerwünschte Effekte wurden nicht beobachtet. ob

\section{Kurz notiert}

\section{Xanthelasmen als Hinweis auf Herzgefahr}

Xanthelasmen an Augenlidern, eigentlich nur kosmetisch störend und aus einer Cholesterolansammlung bestehend, können ein Hinweis auf ein erhöhtes Risiko für eine ischämische Herzkrankheit oder einen Herzinfarkt sein. Das sagte Dr. Mette Christoffersen aus Kopenhagen in Chicago. Gefunden wurde die Assoziation in epidemiologischen Studien. Die Assoziation bestand unabhängig von erhöhtem LDL.

\section{Koronarkalk sagt mehr als CRP-Test}

Erhöhte CRP-Spiegel waren bei normalem LDL-Wert in der JUPITER-Studie ein Marker für ein verstärktes Gefäßrisiko und damit eine Indikation zur Statintherapie. Werden die Patienten zusätzlich auf Koronarkalk untersucht, konnte eine noch gröBere Treffsicherheit bei der Risikobewertung erzielt werden, erläuterte Dr. Michael Blaha aus Baltimore beim US-Kardiologenkongress - auf Basis einer retrospektiven Analyse.

\section{RK}

\section{CRP-Debatte: Marker oder auch nicht?}

Ob erhöhte hochsensitive CRP-Werte in der Klinik schon eine Indikation für eine Statintherapie sind, daran haben Experten erhebliche Bedenken angemeldet. CRP-Messungen gehörten auch nicht in die Routine der Risikobestimmung, da sie keinen stärkeren Aussagewert als die Bestimmung der klassischen Risikofaktoren einschließlich der Blutdruckmessung haben, erläuterte Prof. Peter Sever aus London beim AHA. 